\title{
Effect of Surface Coating on the Photocatalytic Function of Hybrid CdS-Au Nanorods
}

\author{
Yuval Ben-Shahar, Francesco Scotognella, Nir Waiskopf, Ilka Kriegel, \\ Stefano Dal Conte, Giulio Cerullo, and Uri Banin*
}

$\boldsymbol{H}_{y b r i d}$ semiconductor-metal nanoparticles are interesting materials for use as photocatalysts due to their tunable properties and chemical processibility. Their function in the evolution of hydrogen in photocatalytic water splitting is the subject of intense current investigation. Here, the effects of the surface coatings on the photocatalytic function are studied, with Au-tipped CdS nanorods as a model hybrid nanoparticle system. Kinetic measurements of the hydrogen evolution rate following photocatalytic water reduction are performed on similar nanoparticles but with different surface coatings, including various types of thiolated alkyl ligands and different polymer coatings. The apparent hydrogen evolution quantum yields are found to strongly depend on the surface coating. The lowest yields are observed for thiolated alkyl ligands. Intermediate values are obtained with Lglutathione and poly(styrene-co-maleic anhydride) polymer coatings. The highest efficiency is obtained for polyethylenimine (PEI) polymer coating. These pronounced differences in the photocatalytic eff ciencies are correlated with ultrafast transient absorption spectroscopy measurements, which show a faster bleach recovery for the PEI-coated hybrid nanoparticles, consistent with faster and more effi cient charge separation. These differences are primarily attributed to the effects of surface passivation by the different coatings affecting the surface trapping of charge carriers that compete with effective charge separation required for the photocatalysis. Further support of this assignment is provided from steady-state emission and time-resolved spectral measurements, performed on related strongly fl uorescing $\mathrm{CdSe} / \mathrm{CdS}$ nanorods. The control and understanding of the effect of the surface coating of the hybrid nanosystems on the photocatalytic processes is of importance for the potential application of hybrid nanoparticles as photocatalysts.

\section{Introduction}

Conversion of renewable solar energy to chemical energy stored in a fuel, in particular hydrogen, is attracting renewed attention. ${ }^{[1-3]}$ Hydrogen can be generated via the photocatalytic water splitting reaction and the possibility of using highly controlled hybrid semiconductor-metal nanoparticles (NPs) as photocatalysts has been addressed recently. ${ }^{[4-8]}$ Such hybrid NPs (HNPs) exhibit light induced spatial charge separation at the semiconductor-metal interface. The separated charge carriers can be harnessed for performing redox

\author{
Y. Ben-Shahar, N. Waiskopf, U. Banin \\ The Institute of Chemistry and \\ Center for Nanoscience and Nanotechnology \\ The Hebrew University of Jerusalem \\ Jerusalem 91904, Israel \\ E-mail: Uri.Banin@mail.huji.ac.il \\ F. Scotognella, I. Kriegel, S. Dal Conte, G. Cerullo \\ IFN-CNR, Dipartimento di Fisica \\ Politecnico di Milano \\ Milan 20133, Italy
}

This is the peer reviewed version of the following article: "Effect of surface coating on the photocatalytic function of hybrid CdS-Au nanorods" (Small, 2015. 11:4, 462-471), which has been published in final form at https://doi.org/10.1002/smll.201402262. This article may be used for non-commercial purposes in accordance with Wiley Terms and Conditions for Use of Self-Archived Versions 
chemistry which can also be applied towards water splitting to generate hydrogen. A unique attribute of the HNPs, is the highly developed control over various characteristics important for tailoring their photocatalytic function. The semiconductor part can be adjusted for optimal solar spectral coverage and also designed to enhance the charge separation efficiency by tailoring its composition and shape. ${ }^{[9,10]}$ In addition, the co-catalyst sites can be controlled and combinations of noble-metals and transition-metal oxides and sulfides were investigated, ${ }^{[11-15]}$ as well as the use of enzymatic co-catalysts. ${ }^{[16,17]}$ The dynamics of the charge separation processes and the photocatalytic function were also studied to provide further understanding of the related mechanisms. ${ }^{[18-21]}$

An additional important factor for the photocatalytic function of the colloidal HNPs, which has not been systematically studied yet, is the effect of the surface coating. The surface coating needs to provide colloidal stability in water for the photocatalyst NPs, while at the same time maintain good accessibility to the active surface sites from where charge carriers need to be transferred for the reaction. The surface coating can also affect trapping of light generated charge carriers at surface defects which may reduce the photocatalytic efficiency.

Here we study the effects of the surface coating on the photocatalytic function of hybrid metal-semiconductor nanoparticles. We use a well-controlled model nanoparticle system of hybrid $\mathrm{Au}$ tipped $\mathrm{CdS}$ nanorods allowing for a systematic study that isolates the effect of the surface coating. ${ }^{[22,23]}$ The stabilization of the hybrid nanorods in water was achieved by several surface coating strategies, which were adopted from the highly evolved work on rendering semiconductor nanocrystals water soluble for their use in biological tagging applications. $^{[24-26]}$ Specifically, a comparison between different types of thiolated-alkyl ligands and polymer coatings was carried out to study the effect of the surface coating on the photocatalytic water reduction reaction. Ultrafast transient absorption spectroscopy measurements were also performed to follow the surface coating effect on the charge separation process at the metal-semiconductor interface. Comparing these observations with steady-state and timeresolved emission spectra measurements on related highly emitting $\mathrm{CdSe} / \mathrm{CdS}$ heterostructured nanorods allows us to propose and establish a mechanism for the observed strong surface effect, related to the surface passivation impacting the trapping of charge carriers.

\section{Results and Discussion}

\subsection{CdS-Au HNP Synthesis and Phase Transfer to Water}

The synthesis of the HNP model system was carried out in consecutive steps. First, $\mathrm{CdS}$ or seeded $\mathrm{CdSe} / \mathrm{CdS}$ nanorods were synthesized by a seeded-growth method. ${ }^{[27,28]}$ Next, selective metal growth on the semiconductor nanorod apex was carried out under dark conditions and at room temperature (see SI). ${ }^{[29,30]}$ Figure 1a shows a transmission electron microscopy (TEM) image of the hybrid CdS-Au nanorods. Selective single $\mathrm{Au}$ tip growth on one apex of all
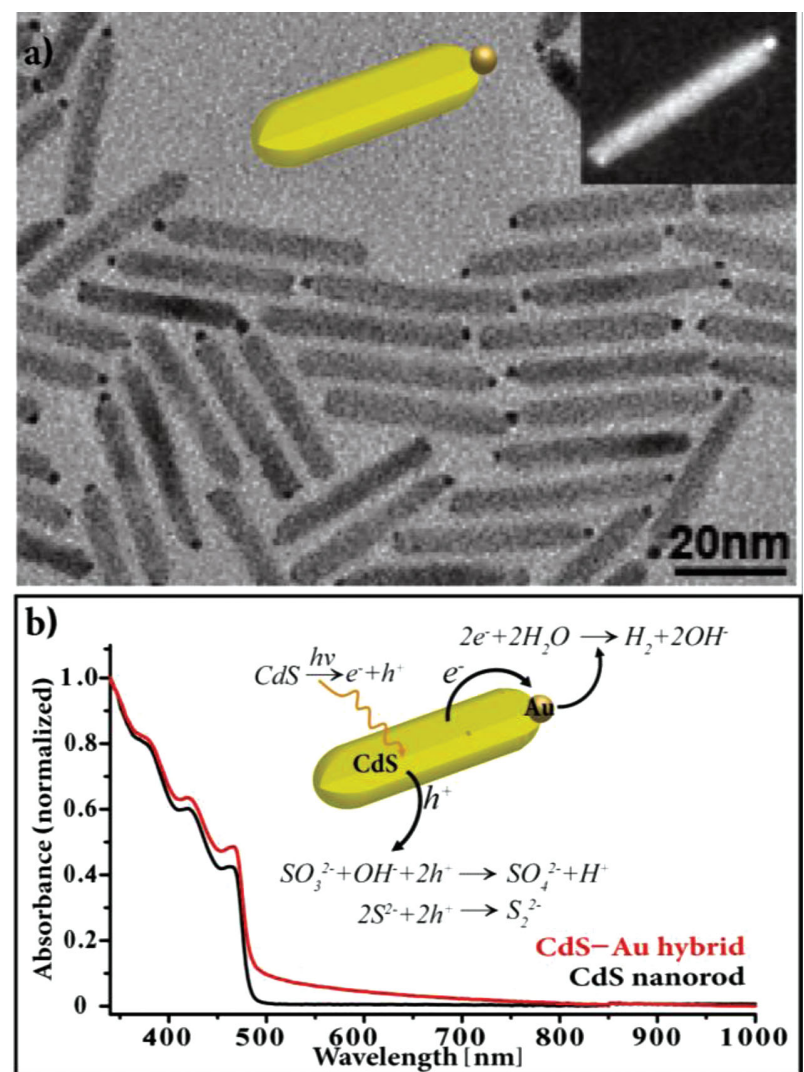

Figure 1. (a) TEM and HAADF-STEM (inset) images of CdS-Au HNPs $(37 \times 5 \mathrm{~nm})$ with $1.8 \pm 0.2 \mathrm{~nm}$ Au tip size. (b) Absorbance spectra of CdS NRs (black line) and CdS-Au HNPs (red line). Inset shows a scheme of the photocatalysis reaction in the presence of hole scavengers.

the nanorods providing a matchstick-like structure is seen. The single small metal tips were of diameter of $1.8 \mathrm{~nm}$ with a good size distribution ( $12 \%$, see SI Figure S1 for the sizing histograms). Further characterization of the hybrid nanorods was performed using high angle annular dark-field scanning TEM (HAADF-STEM) and a typical image is shown in the inset of Figure 1a. The method provides Z-contrast imaging, and the small Au tip is clearly seen at the apex.

Figure $1 \mathrm{~b}$ compares the absorption spectra of the bare $\mathrm{CdS}$ nanorods and the hybrid CdS-Au nanorods. Both spectra exhibit a similar sharp rise at $478 \mathrm{~nm}$ related to the onset of the CdS nanorods absorption that is blue shifted compared to the bulk CdS band gap because of the quantum confinement related to the nanoscale diameter of the rods. Several absorption features are seen to the blue of the onset, related to the band gap and to higher excited optical transitions of the CdS rods which signify the good size monodispersity of the sample. In the hybrid CdS-Au nanorod spectrum, a tail towards the red is seen related to the contribution of the small Au tip. This is consistent with the featureless absorption in this spectral range observed for $1.7 \mathrm{~nm}$ Au clusters. ${ }^{[31]}$ The absorbance in the CdS region is increased compared to that of the bare CdS rod, related also to the contribution of the Au tip.

For the study of the effect of the surface on the photocatalytic activity of the HNPs, phase transfer to water was performed using several different strategies modified from 


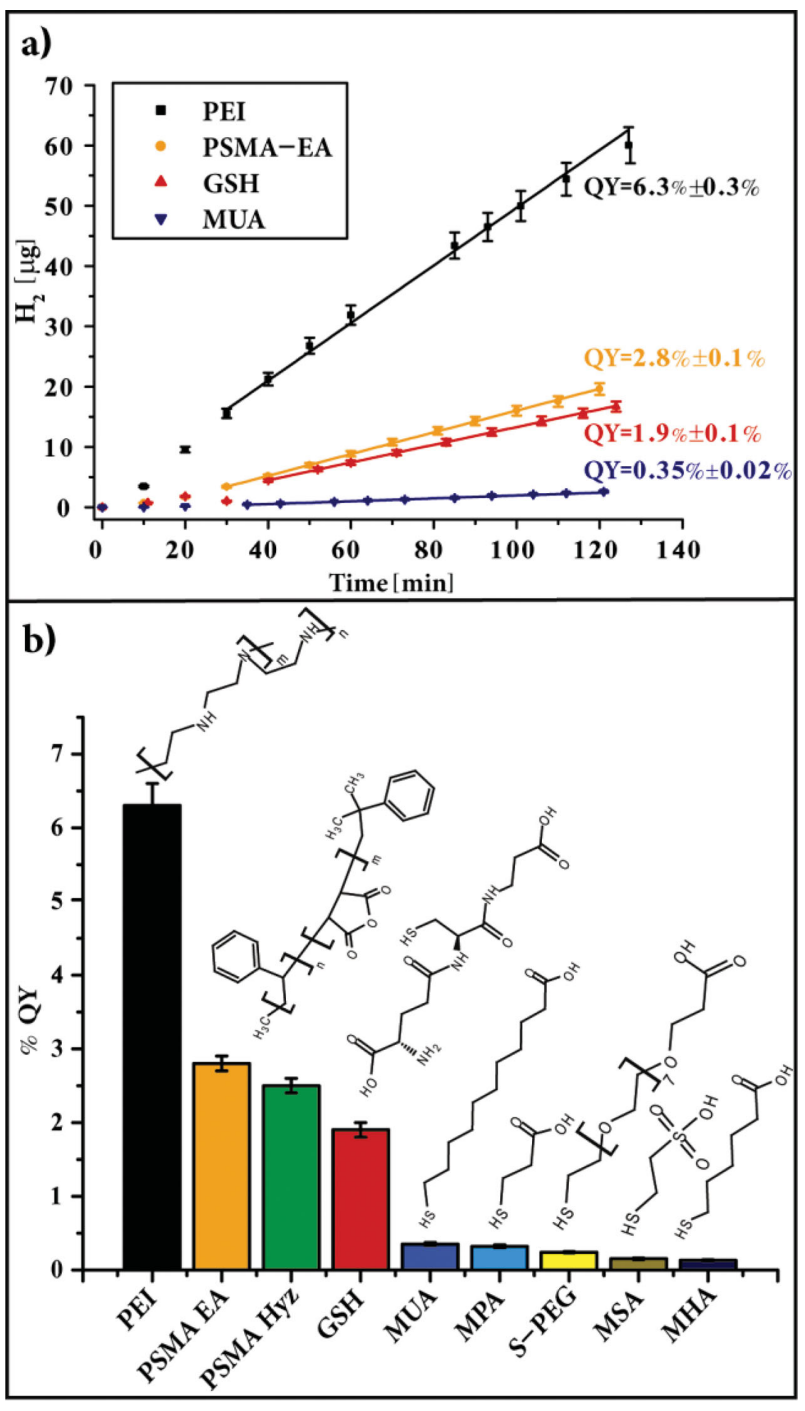

Figure 2. (a) Kinetic hydrogen evolution measurements by CdS-Au HNPs for different surface coatings. Straight lines represent the linear fits from which the \%QY was extracted. (b) Apparent photocatalysis \%QY values for a wide range of surface coatings including thiolated alkyl ligands, GSH, and polymer coating. PEI exhibits the highest QY.

the water transfer reactions developed for biological applications of semiconductor nanocrystals (Figure 2b). ${ }^{[24,32,33]}$ First, ligand exchange of the hydrophobic phosphonic acid and alkylamine ligands was performed to different types of thiolated alkyl ligands. A first ligand type that was studied is that of mercaptocarboxylic acids with different chain lengths comprising of mercaptoundecanoic acid (MUA), mercaptohexanoic acid (MHA) and mercaptopropionic acid (MPA). An additional ligand was L-glutathione (GSH), a tripeptide with two carboxylate and one amine end groups per ligand, which hence holds higher charge in basic solution. ${ }^{[24]}$ Mercaptosulfonic acid (MSA) was studied as an additional type with a different charged end group. Another member of this group of thiol bound ligands was O-(2-carboxyethyl)-O'-(2mercaptoethyl)heptaethylene glycol (S-PEG) providing a fairly long oligomer chain.

An additional phase transfer strategy that was performed is polymer encapsulation that is known to provide very good colloidal stability and environmental protection. This was carried out using polyethylenimine (PEI) ${ }^{[25]}$ which binds with amine groups to the surface of the nanorod through ligand exchange. Another approach using an amphiphilic agent that maintains the original nanorod surface coating by encapsulating it and presenting hydrophilic end groups to the solution, was carried out using poly(styrene-co-maleic anhydride) (PSMA). ${ }^{[26]}$

\subsection{Hydrogen Evolution Kinetics and Photocatalytic Quantum Yield Measurements}

The different surface coatings were used in a comparative study to examine the surface effect on the photocatalytic rate and efficiency. The photocatalytic effect comprised of the reduction of water to produce hydrogen as depicted schematically in the inset of Figure 1b. Aqueous solutions of the HNPs in the presence of $\mathrm{Na}_{2} \mathrm{~S}-9 \mathrm{H}_{2} \mathrm{O}$ and $\mathrm{Na}_{2} \mathrm{SO}_{3}$, acting as sacrificial hole scavengers, ${ }^{[11]}$ were irradiated with a $40 \mathrm{~mW}$ $405 \mathrm{~nm}$ laser. An optical density of $\sim 1$ at $405 \mathrm{~nm}$ was used. The amount of hydrogen gas produced was determined using a gas chromatograph (GC) equipped with a thermal conductivity detector (see experimental section).

Kinetic measurements of hydrogen evolution are presented in Figure 2a. For all types of surface coatings, after an induction time, the amount of hydrogen product increases linearly with time, during the $2 \mathrm{~h}$ of illumination. The induction time is attributed to the solubility of hydrogen gas in the aqueous solution, and only after the solution is saturated all the evolving hydrogen gas is extracted to the head space of the reaction vessel. Accordingly, linear fitting for hydrogen evolution rate was performed for times longer than $30 \mathrm{~min}$. The trace for MUA is representative of the behavior of the thiolated-alkyl ligands and shows low rate of hydrogen production, while GSH, which differs from the latter by additional carboxylic and amine groups, shows significantly higher rate. In comparison to these rates, polymer coated hybrids provide even higher rates, as is shown by PSMA. The highest rate was detected for PEI coated hybrids, which is one order of magnitude higher than MUA and 3 and 2 times higher than GSH and PSMA, respectively.

The measured rates were used to calculate the apparent quantum yield (\%QY), defined as

$\% Q Y=\frac{n_{e}}{n_{p}} * 100 \%=\frac{2 n_{H_{2}}}{n_{p}} * 100 \%$

where $n_{e}$ is the number of electrons participating in reduction reaction, $n_{p}$ is the number of photons absorbed, and $\mathrm{H}_{2}$ is the number of hydrogen molecules formed and measured by the GC analysis. $n_{p}$ was extracted from the measured illumination intensity and irradiation time, taking into account the solution optical density at the irradiation wavelength, typically $\mathrm{OD}=1$. The $\% \mathrm{QY}$ are shown for the different surface coatings in Figure 2b. All thiolated-alkyl ligands, with one carboxylic group and different lengths of the alkyl chain, had a QY of less than $1 \%$, while GSH reached almost $2 \%$ QY. With correlation to the kinetic observation, polymer coated 
systems have higher \%QY. PSMA treated either with ethanolamine or hydrazine for generating amphiphilic behavior, [26] shows similar values of $2.5 \%$ QY. As can be seen, the highest QY was observed for PEI coating, up to $6.3 \%$. In addition, rate measurement at $1 \mathrm{SUN}$ intensity with a solar simulator setup for hybrids with PEI coating showed high hydrogen evolution rate of $2.94 \times 10^{-5} \mathrm{~g} \mathrm{~h}^{-1}$ (Figure S5). Repetitive measurements on several similar hybrid systems showed the same hydrogen evolution kinetics and efficiency trends, while control experiments on CdS rods without the metal had negligible \%QY (Figure S3). These results demonstrate the critical role of the HNPs surface chemistry on its photocatalytic performance.

\subsection{Mechanism of Surface Coating Effect on Photocatalysis}

We next consider the possible mechanisms for the strong effects of the surface coating on the photocatalytic performance. First, aggregation effects due to the differences in the colloidal stabilization by the various surface coatings, in particular in the presence of the hole scavengers which induce a salting-out effect, may play a role. To minimize aggregation due to salting out, the reported measurements were done in relatively low salt concentration, in which the different surface-coated systems were apparently colloidally stable. Even upon reducing the hole scavenger concentration further, the same trend of \%QY dependence on surface coating was maintained. Therefore, colloidal stability is not the main cause for the difference in this case, although the polymer coatings are also advantageous in this sense and are effective also in high salt concentrations.

An additional effect considered is the nature of the diffuse electric double layer. While the surface of nanorods coated by PEI is positively charged, the thiolated carboxylic ligands are typically negatively charged. Since the rate and efficiency of the photocatalytic reaction depend, among other factors, on effective extraction of the hole out of the system, high accessibility of the hole scavenger to the nanorod surface is required. Given that the hole scavengers used above are polysulfides which are negatively charged, it may be expected that they will be repelled from the surface with the negative thiolated ligands, while surface coating by PEI will attract the sulfide ions and thus may increase the rate of hole neutralization. However, photocatalytic experiments done with a non-charged hole scavenger, ethanol, showed the same trend for the different surfaces, with a decrease in the reaction rates leading to lower \%QY for the PEI $(2.2 \%)$ as well as for GSH $(0.3 \%)$ (Figure S4). In fact, it is seen that for ethanol as hole scavenger GSH representing charged thiolated ligands showed a significantly larger decrease in \%QY in comparison to the decrease with PEI coating despite the absence of the above repulsion effect. We note that control experiments with PEI coated hybrid nanorods but without hole scavengers still provided a measurable small $\%$ QY of $\sim 0.5 \%$ for hydrogen evolution, signifying that PEI indeed may act also as a donor of electrons to the rod. ${ }^{[34]}$ Yet, the effect of hole scavenging by the PEI is expected to be small in the presence of the polysulfide hole scavengers which dominate the hole extraction once they are added.

We also considered the effect of photostability which may contribute to the enhancement of the photocatalytic activity by PEI coated hybrid nanorods while explaining the low efficiency in the alkyl thiol coatings. As has been shown by Nie et al., nanoparticles stabilized by thiolated ligands, such as MPA, undergo photooxidation much more rapidly than PEI coated nanoparticles. ${ }^{[35]}$ This phenomenon was explained by the behavior of the defect sites on the surface after illumination leading to accumulation of photo-generated holes. In presence of oxygen, the deprotonated thiol end groups bound to the surface react to form disulfides as the first stage of the photooxidation. Later, the nanocrystal itself undergoes photooxidation and eventually the nanocrystals precipitated out of the solution. ${ }^{[36]}$ However, in our case, the presence of sulfide and sulfite hole scavengers, and the use of inert atmosphere with little amount of oxygen during the photocatalytic measurements, reduce the influence of this parameter as indeed seen from the linear dependence of the hydrogen evolution with time.

In light of the above discussion, an additional possible effect of the surface coatings on the photocatalytic activity, is the degree of chemical and electronic surface passivation. Electron-hole charge separation across the semiconductormetal interface competes effectively with the direct electron-hole recombination as evidenced by the fluorescence quenching in the HNPs and by time resolved study. ${ }^{[37}$ An additional competing non-radiative route is trapping of the charge carriers in sub-gap energy levels, which exist due to defect sites, typically present on the semiconductor rod surface. Although surface traps can provide long-lived charge carriers available for the reduction reaction, as reported previously ${ }^{[12,13]}$ and in our control measurements (Figure S3), $\mathrm{CdS}$ nanorods without $\mathrm{Au}$ tips show a negligible hydrogen reduction activity which clearly demonstrates the important role of the metal co-catalyst. To further investigate the effect of surface coating on the charge separation dynamics in the hybrid nanoparticles, ultrafast transient absorption spectroscopy measurements were performed as described next.

\subsection{Ultrafast Transient Absorption Measurements}

Further direct evidence for the significant effect of surface coating on charge separation in the hybrid nanoparticles was provided from ultrafast transient absorption (TA) measurements. These were performed on CdS-Au nanorods with $\mathrm{Au}$ domain of $3 \mathrm{~nm}$ in diameter. A comparison of the time resolved TA spectra and dynamics between three different surface coatings - PEI, GSH and MUA, on the same HNPs is presented in Figure 3. Following $450 \mathrm{~nm}$ optical excitation and formation of excited electron-hole pairs, the TA spectra of all three surface coated hybrids reveal a feature of bleach formation around $450 \mathrm{~nm}$ attributed to electron excitation at the first exciton feature of the CdS part of the hybrid nanoparticle. ${ }^{[20]}$ In addition, a broad bleach feature is seen at $540 \mathrm{~nm}$. This feature is related to the plasmon response of the $\mathrm{Au}$ tip part of the HNPs. It exhibits rapid decay with 


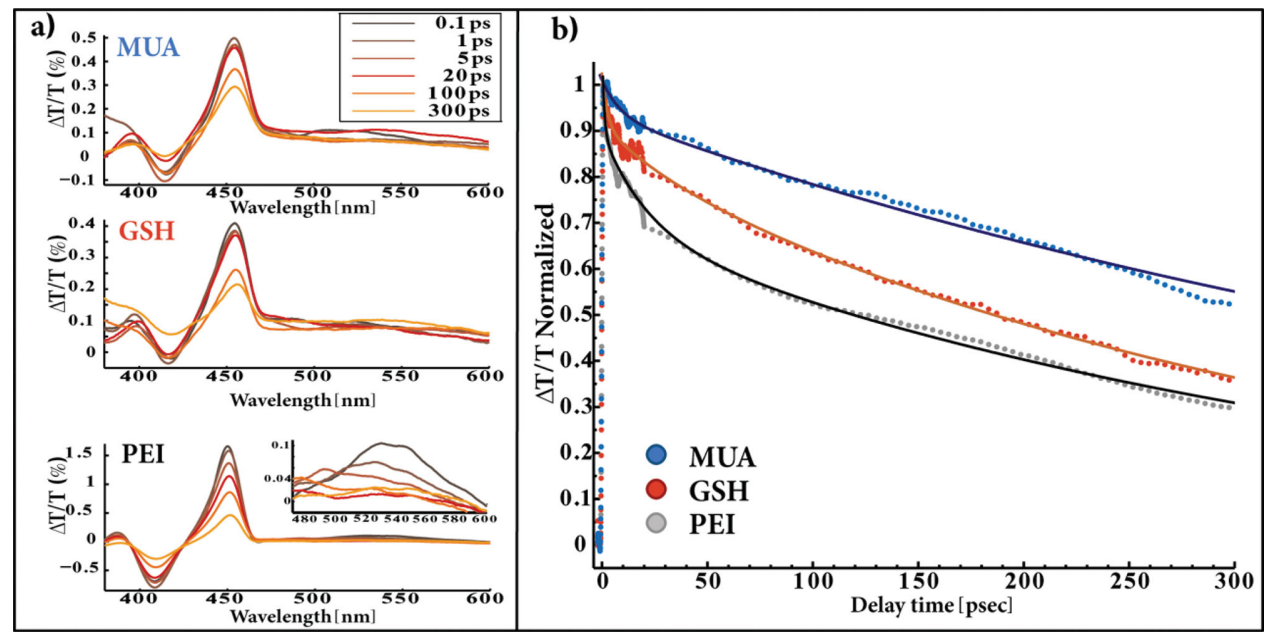

Figure 3. (a) Transient absorption spectra of CdS-Au HNPs for different surface coatings at 450nm excitation. Expansion of the Au plasmon region for the PEI coating are presented in an inset (lower left frame). (b) Normalized transient absorption kinetics of the bleach recovery at $460 \mathrm{~nm}$, corresponding to the first excitonic transition of the CdS nanorod component, along with exponential fits (continuous lines) for CdS-Au HNPs with different surface coatings. Blue color - MUA coating, red -GSH, black - PEI.

timescale of $\sim 2-3$ ps. (Figure $3 a$ inset). This type of recovery of the plasmon component of the spectrum is related to rapid relaxation of hot electrons consistent with previous reports on colloidal gold nanoparticles. ${ }^{[38]}$ Additional details of the TA measurements are given in the Supporting Information.

Normalized TA kinetics for the bleach recovery in the spectral region of the CdS band gap exciton are presented in Figure $3 \mathrm{~b}$ for the three different surface coatings. The observed decays exhibit a fast recovery component followed by slower decay components. TA measurements of the same CdS rods, but without metal domain, shows slower decay times relative to the HNPs (Figure S6). As discussed in previous works, the fast recovery of the excitonic bleach in HNPs is attributed to the charge transfer of the excited electrons from the semiconductor nanorod conduction band edge to the Fermi level of the metal domain. ${ }^{[20,39,40]}$ Indeed, the absence of the fast route of electron removal in the CdS rods case, where charge separation does not take place, is consistent with this interpretation.

Comparison between the TA dynamics of HNPs with different surface coatings at $460 \mathrm{~nm}$ reveals two substantial and related differences. First, fastest charge transfer dynamics is seen in the case of the PEI coated HNPs, slower with GSH, and the slowest with MUA passivated particles as revealed from the experimental measured half-lives of $100 \mathrm{ps}$ for PEI and $160 \mathrm{ps}$ and $330 \mathrm{ps}$ for GSH and MUA, respectively. A related second difference is that the decay amplitude over the measurement timescale in these experiments is also largest in the PEI coated system. The trend of decay dynamics is consistent with rapid electron transfer with PEI coating, slower with GSH, and least effective electron transfer for MUA coated HNPs.

These two differences are also reflected by tri-exponential model fitting parameters for the bleach decay curves of the CdS-Au HNPs (Table S1). The faster decay components relevant for electron transfer to the metal domain and their relative amplitudes, exhibit a similar trend between the different coatings. PEI coated HNPs display the fastest decay constants, and largest amplitudes for these components. GSH has intermediate slower timescale and smaller decay amplitudes, while MUA shows the slowest times and smallest amplitudes. More detailed explanations are provided in the Supporting Information Online, along with fit parameters for the TA decays of CdS rods with the different coatings (table S2).

These differences are attributed to improved surface passivation of the particle surface, therefore decreasing the available hole trapping sites. Trapping of holes leads also to slower electron transfer due to the electron-trapped hole Coulomb interactions. ${ }^{[21]}$ To avoid this loss route through surface trapping, surface defects must be passivated. This is particularly important for the nanorod systems since optical excitation in the semiconductor leads to electron-hole pair formation along the CdS rod and there is relatively large surface area for carrier trapping. This gains further emphasis considering that the long rod axis is significantly larger than the typical electron-hole extent in CdS for which the exciton Bohr radius is $2.8 \mathrm{~nm} .{ }^{[41]}$ This kind of model was invoked previously by Berr et al. in the case of photocatalytic Pt decorated $\mathrm{CdS}$ rods. ${ }^{[21]}$

This trend in charge separation rates for the different surface coatings is consistent with their respective photocatalytic efficiencies. The MUA coated rods manifest the slowest charge separation rate and smallest amplitude, and also exhibit the lowest photocatalytic efficiency in line with facile hole trapping, GSH manifests intermediate behavior in all these aspects. PEI, with the fastest charge transfer rate and highest amplitude, also exhibits the most efficient charge separation behavior. Effective Surface passivation is therefore critical in the photocatalysis process, to minimize the trapping of the charge carriers, which prevents the efficient charge transfer across the semiconductor-metal interface.

\subsection{Steady-State Emission and Time-Resolved Measurements}

To further complement the effect of the different surface coatings, we also performed fluorescence intensity and 
lifetime measurements that are also sensitive to carrier trapping in the rods surface. Since the HNPs do not show significant fluorescence due to the quenching by the charge transfer to the metal domain, and since the $\mathrm{CdS}$ nanorods have low band gap fluorescence as well, seeded $\mathrm{CdSe} / \mathrm{CdS}$ core/shell nanorods with similar size and surface coatings to the model system were used. The seeded rods are synthesized by a method similar to that of the CdS rods, using a CdSe seed instead of the CdS seed used in the latter. The surface characteristics of the CdS and seeded rods systems are also very similar. However, such $\mathrm{CdSe} / \mathrm{CdS}$ seeded nanorods provide high fluorescence intensity due to the presence of the CdSe seed that is well passivated by the CdS rod-shell. ${ }^{[28]}$

Two different seeded NRs were studied, with different CdSe seed diameters of $2.1 \mathrm{~nm}$ and $4.3 \mathrm{~nm}$. The seed size affects the extent of the electron wavefunction, but has a much smaller effect on the hole wavefunction of the band gap exciton which remains well-localized in the CdSe seed in both systems (Figure 4). Therefore, in the larger seed, the electron and hole are both essentially localized within the CdSe core (type-I behavior). ${ }^{[28]}$ For the smaller seed, due to the quantum confinement, the electron state lies above the $\mathrm{CdSe} / \mathrm{CdS}$ conduction-band barrier leading to stronger delocalization of the electron into the $\mathrm{CdS}$ rod region, while the hole remains localized in the CdSe seed (quasi type-II behavior). ${ }^{[42]}$ However, $\mathrm{Wu}$ et al. recently reported three different relaxation locations for quasi type-II $\mathrm{CdSe} / \mathrm{CdS}$ nanorod upon $400 \mathrm{~nm}$ excitation, including in the CdS shell region with $46 \%$ probability. This means that excitation above the CdS shell band gap may lead to hole trapping in the CdS surface followed by partial co-localization of the electron near such a trap site. ${ }^{[43]}$ In this respect, in the quasi type-II system, both delocalization of the electron wavefunction and the formation of charge carrier pairs near the surface, leads to higher sensitivity to the surface coating since throughout the relatively long lifetime of the excited state, surface trapping of the electron leading to a non-radiative route can also occur effectively.

The structural and optical characterization of the seeded rods is presented in Figure S2 in the Supporting Information. The sizing analysis allowed to extract the rods dimensions for the quasi type-II system $-2.1 \mathrm{~nm} / 27 \times 4 \mathrm{~nm}$ (seed diameter/ rod length $\times$ rod diameter) with emission peak at $551 \mathrm{~nm}$, while the type-I system dimensions are $4.3 \mathrm{~nm} / 30 \times 4.7 \mathrm{~nm}$ with emission peak at $623 \mathrm{~nm}$. The nanorods were transferred to aqueous solution by the same protocols used for the HNPs with various ligands including PEI, PSMA, GSH and MUA.

Steady-state photoluminescence (PL) measurements of the seeded $\mathrm{CdSe} / \mathrm{CdS}$ rods with quasi type-II and type-I character are presented in the insets of Figure $4 \mathrm{a}$ and b, respectively. In both seeded rod systems, the surface coating has a significant effect on the PL intensity. MUA coating, representative of the family of the thiolated alkyl ligands, leads to the largest decrease in PL intensity. GSH and PSMA coatings exhibit intermediate PL intensity values. The highest PL intensity, even higher relative to the PL of the TOPO coated rods in the organic solution, is observed for PEI coating. These trends in PL intensities and fluorescence quantum yield (Table S5) are consistent with the trend of the photocatalytic
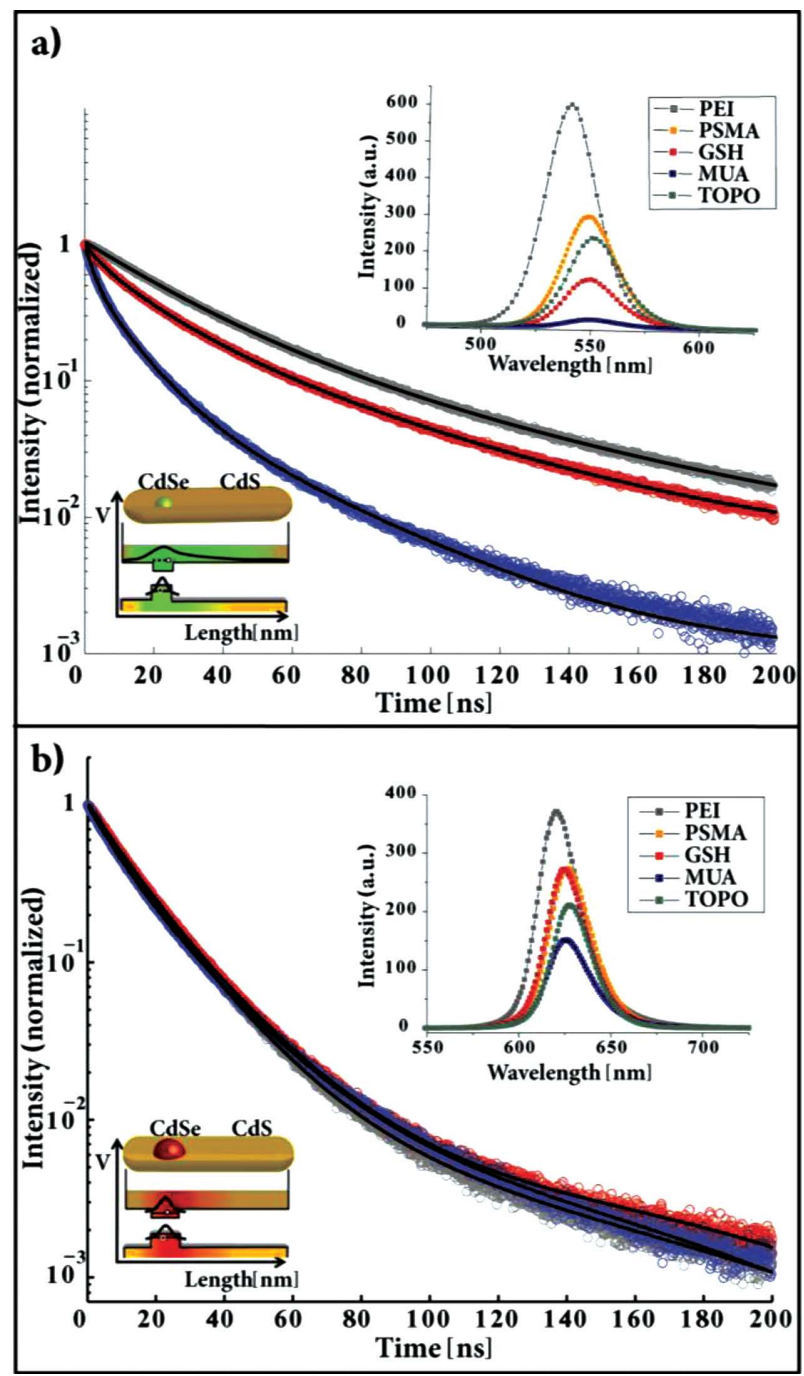

Figure 4. Steady-state emission spectra (upper right insets) and time-resolved fluorescence decay curves with three exponential fits (continuous black lines) for $\mathrm{CdSe} / \mathrm{CdS}$ core/shell seeded nanorods with seed size of $2.1 \mathrm{~nm}$ (a) and $4.3 \mathrm{~nm}$ (b). Effective lifetime values were obtained from $\tau_{1 / e}$. The lower left insets show a scheme of the potential profile and electron and hole wavefunction extent for each system, with quasi type-II behavior in (a), and type-I behavior in (b). Blue color - MUA coating, red - GSH, gray - PEI.

\%QY for the different surface coatings, with low efficiencies seen in alkyl thiolated ligands, intermediate values for PSMA and GSH, and the highest efficiency observed for the PEI. Moreover, they are also consistent with the electron transfer rates extracted from the TA study as discussed above.

Comparison between the two systems shows that the relative change in the intensity is much more pronounced in the quasi type-II system compared to the type-I system. In correspondence to the spectral studies, lifetime measurements for the quasi type-II system reveal longer effective fluorescence lifetime $\left(\tau_{1 / e}\right)$ of PEI coated nanorods (31ns) relative to GSH (19 ns) and MUA (7 ns) surface ligands (Figure 4a). Type-I system lifetime measurements are nearly similar and do not exhibit the same pronounced effect as in the quasi type-II system (Figure 4b) with measured effective lifetimes of 14 ns for both PEI and GSH, and 12 ns for MUA. 
Additional information including linear display and multiexponential fi tting is available in the Supporting Information (Figures S7, S8).

The PL intensity and lifetime are dictated by the competition between the non-radiative and radiative decay routes. As noted above, the two systems differ essentially by their sensitivity to the surface due to their energy profile. Accordingly, in the quasi type-II case, the dependence of the fluorescence intensity on the surface coating is correlated with the change in fl uorescence lifetime, consistent with MUA surface coating presenting the most signifi cant surface trapping, GSH providing intermediate passivation, and PEI offering the best passivation of surface traps for electrons. In the type-I case on the other hand, the little change in lifetime signifies the rapid relaxation of the excited electron into the CdSe seed, which exceeds the charged carrier's surface trapping time. For electron-hole pairs that localized in the seed, a similar decay rate can be expected as the surface effects are diminished. The effect of the surface coating on the fluorescence intensity accompanied by very small change in lifetimes, suggests a change in the proportion of emitting versus nonemitting rods, due to trapping of the carries prior to carrier localization in the seed.

The fl uorescence study and the related explanation are consistent with the TA observations and the trend of the photocatalytic effi ciency dependence observed for the CdS-Au nanorods with different surface coatings.

To further verify the relevance of the surface effect directly also for photocatalysis with seeded rods, Au tips were grown on the quasi type-II $\mathrm{CdSe} / \mathrm{CdS}$ nanorods and their TEM image and absorbance are presented in Figure5. The comparison of the hydrogen evolution kinetics and \%QY for these nanorods with different surface coatings is presented in Figure 5c. The results closely follow the trends observed for the CdS-Au hybrids where MUA has low efficiency, GSH shows intermediate effi ciency and PEI provides the highest hydrogen evolution \%QY. This is consistent with the photocatalytic effi ciency behavior of the CdS-Au hybrid NP's with the different surface coatings pointing out to the main effect of surface passivation which upon reduction of hole trapping, allows for enhancing the charge separation and photocatalytic efficiency.

$\mathrm{T}$ he observed longer excited state lifetime, due to improved passivation with PEI, is consistent with the improvement in the catalytic activity. This infl uence of longlived charged carriers on the photocatalytic efficiencies of hydrogen evolution is consistent with the work of Thibert et al. on related nanocrystal systems. ${ }^{[44]}$ They reported that the photocatalytic effi ciency of CdSe quantum dots was very small, while overcoating with thin CdS shell provided a significant improvement. This was attributed to passivation of deep traps that suppressed the possibility for hydrogen generation in the CdSe cores only system. This enhanced photocatalysis is also accompanied by the enhanced PL performance of the CdSe/CdS core/shell QDs suppressing deep trap emission.

Similar to the photocatalytic system charge separation demands, photovoltaic devices with nanocrystal building blocks also require effi cient charge separation and transport of charge carriers. Previous works have demonstrated the

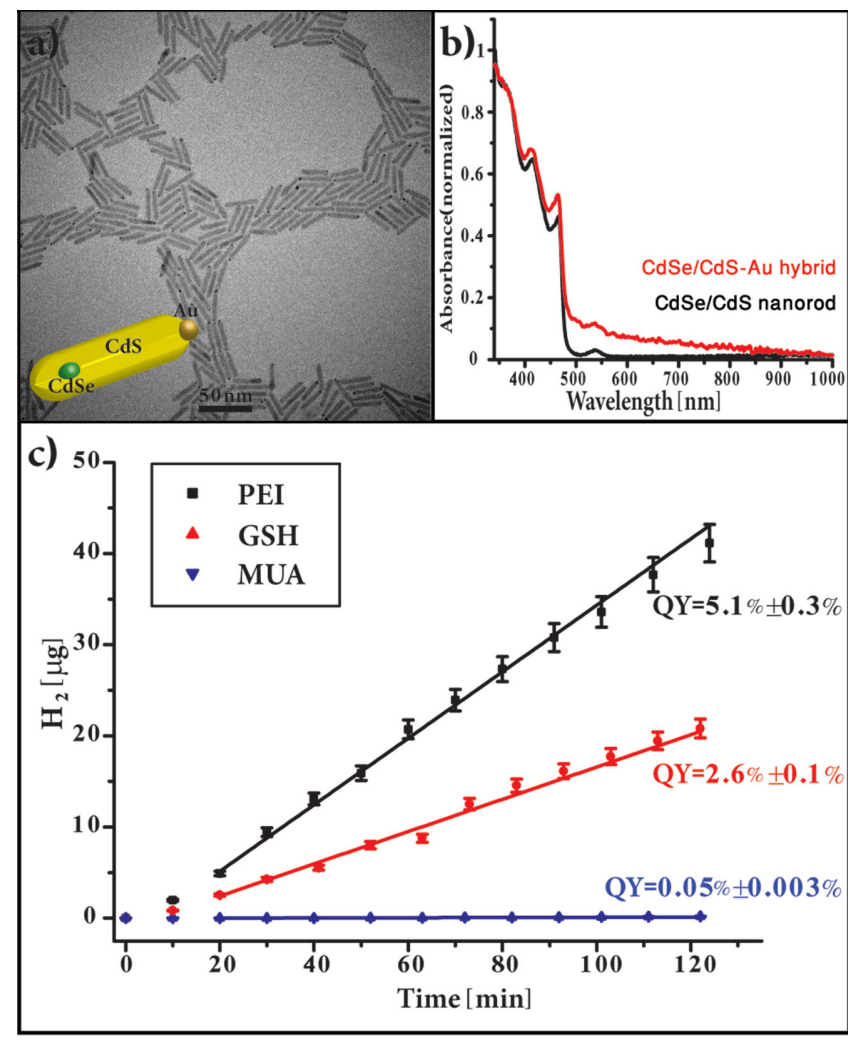

Figure 5. Photocatalytic hydrogen evolution experiments for CdSe/ CdS core/shell seeded nanorods with seed size of $2.1 \mathrm{~nm}$ and overall dimensions $27 \times 4.1 \mathrm{~nm}$ (quasi type-II case shown in Figure $3 \mathrm{a}$ ) and Au tip size of $1.8 \mathrm{~nm}$ with different surface coatings. (a) TEM image. (b) Absorption spectra of CdSe/CdS NRs (black line) and CdSe/CdS-Au HNPs (red line). (c) Kinetic hydrogen evolution measurements for CdSe/ CdS-Au HNPs with different surface coating. Straight lines represent the linear fits from which the \%QY was extracted.

importance of suppressing surface defects which lead to a competing loss-decay route. Improved photovoltaic performance was achieved after surface treatment that suppressed deep traps on the nanocrystal surface and prevented the excited charge carriers trapping in surface states. ${ }^{[45,46]}$

\section{Conclusion}

We have studied the effect of surface coating on the photocatalytic efficiency of hybrid semiconductor-metal nanoparticles in relation to the light induced water reduction. Thiolated alkyl ligands all show low photocatalytic activity, while improved performance was seen for GSH and polymer coating with PSMA. The best photocatalytic activity was found for PEI-coated HNPs. Correlating the results of photocatalytic measurements with transient absorption spectroscopy and fluorescence intensity and lifetime measurements suggests that the significantly improved photocatalytic properties of the PEI-coated hybrid NPs are mainly related to the effect of passivation of surface traps. In terms of the apparent $\%$ QY results, the previous studies of related systems using $\mathrm{CdS}$ rods of similar dimensions with different co-catalysts, all provided lower apparent \%QYs (up to $4 \%$ ), ${ }^{[9,13]}$ even for $\mathrm{Pt}$ 
co-catalyst which is considered to be an optimal co-catalyst. Therefore, the PEI coating holds promise in providing higher efficiency alongside with high colloidal stability required for the demanding performance of a photocatalyst in solution.

\section{Experimental Section}

Synthesis of CdS Seeds: CdS nanocrystal seeds were synthesized by a modification of a previously reported procedure. ${ }^{[47]}$ cadmium oxide ( $\mathrm{CdO} ; 0.106 \mathrm{~g}$ ), oleic acid (OA; $2.26 \mathrm{~g})$ and 1-octadecene (ODE; $20 \mathrm{~g}$ ) were mixed in a $100 \mathrm{~mL}$ three-neck flask. The mixture was heated to $100{ }^{\circ} \mathrm{C}$ and placed under vacuum for $1 \mathrm{~h}$ followed by purging three times with argon. Under argon atmosphere, the solution was heated to $260{ }^{\circ} \mathrm{C}$ to dissolve the $\mathrm{CdO}$, forming a clear colorless solution. A precursor solution consisting of sulfur $(0.013 \mathrm{~g})$ and ODE $(7 \mathrm{~mL})$ was rapidly injected into the hot solution. The reaction time was typically $90 \mathrm{~s}$ for $\mathrm{CdS}$ seeds with diameter of $\sim 3.1 \mathrm{~nm}$. The reaction was quenched by removing the heating mantle and cooling with fan. The crude reaction mixture was precipitated with acetone followed by centrifugation. For further purification, the particles were dissolved in toluene and the precipitation procedure was repeated several times.

Synthesis of CdS Nanorods: CdS nanorods were synthesized by a modification of a previously reported procedure employing seeded growth. ${ }^{[27]} \mathrm{CdO}$ (0.12 g), trioctylphosphine oxide (TOPO; $6.0 \mathrm{~g}$ ), octadecylphosphonic acid (ODPA; $0.68 \mathrm{~g}$ ) and hexylphosphonic acid (HPA; $0.04 \mathrm{~g}$ ) were mixed in a $100 \mathrm{~mL}$ three-neck flask. The mixture was heated to $100{ }^{\circ} \mathrm{C}$ and placed under vacuum for 1 $\mathrm{h}$ followed by purging three times with argon. Under argon atmosphere, the solution was heated to $350^{\circ} \mathrm{C}$ and at this temperature trioctylphosphine (TOP; $1.5 \mathrm{~mL}$ ) was injected into the hot solution. Next, the solution was heated further to $365^{\circ} \mathrm{C}$, at which a precursor solution of CdS seeds (typically, $3 \times 10^{-8} \mathrm{~mol}$ ) and sulfur in TOP solution $(1.6 \mathrm{~mL} ; 0.075 \mathrm{~g} / \mathrm{mL}$ ) was rapidly injected into the hot solution. The temperature decreased and then recovered within 1-2 $\mathrm{min}$. The reaction time was 8 min for $30 \times 5 \mathrm{~nm}$-sized $\mathrm{CdS}$ rods. The reaction was quenched by removing the heating mantle and cooling with fan. The crude reaction mixture was diluted with toluene. Methanol was added in order to precipitate the nanocrystals and remove excess surfactants. The nanorod samples had narrow size distribution according to their absorption spectra and TEM characterization measurements and statistics.

Synthesis of Hybrid CdS-Au Nanorods: A precursor stock solution consisting of octadecylamine (ODA; $0.042 \mathrm{~g})$, di-dodecylammoniumbromide (DDAB; $0.021 \mathrm{~g}$ ) and $\mathrm{AuCl}_{3}(0.015 \mathrm{mg})$ in toluene $(10 \mathrm{~mL})$ was sonicated for $15 \mathrm{~min}$ to dissolve the $\mathrm{AuCl}_{3}$, and the solution changes color from dark brown to yellow. In order to achieve selective growth of 1.5-1.8 nm gold tips on one apex of the nanorods, the molar ratio of $\mathrm{Au}^{3+} /$ nanorod used was $700-900$ $\mathrm{Au}$ ions per nanorod depending on the specific properties of the rods. Diluted Au growth stock solution was added to $\mathrm{CdS}$ nanorods (typically $\left.\sim 7 \times 10^{-9} \mathrm{~mol}\right)$ in toluene $(20 \mathrm{~mL})$ in $50 \mathrm{ml}$ flask under flowing argon. The solutions are mixed and left for $1 \mathrm{~h}$ in the dark at room temperature. The product HNPs are then washed and precipitated with acetone followed by separation via centrifugation. ${ }^{[48]}$

Phase Transfer: For transfer of the HNPs to water, ligand exchange method and polymer coating method were used. For exchanging the native organic-soluble ligands with the thiolated alkyl ligands the ligand exchange strategy was used ${ }^{[24]}$ in which a stock solution is prepared by adding mercaptoundecanoic acid (MUA;0.1 g) and $\mathrm{KOH}(30 \mathrm{mg}$ ) to methanol (1 mL). Next, $200 \mu \mathrm{L}$ of stock solution is added to hybrid CdS-Au nanorods in chloroform $(1 \mathrm{~mL})$ with an optical density of 1.5 at the CdS first transition and mixed for 1-2 min. Based TDW (pH 11-12) is added to the flocculated solution and after mixing again phase separation appears and the nanorods are extracted from the upper water phase after mild centrifugation. The transfer with the additional ligands mercaptohexanoic acid (MHA), mercaptopropionic acid (MPA), 2-mercaptoethanesulfonic acid (MSA), 0-(2-carboxyethyl)$\mathrm{O}^{\prime}$-(2-mercaptoethyl)heptaethylene glycol and L-glutathione (GSH) was performed by a similar protocol, with adjustments of reagents concentrations.

Polymer coating was done with different polymers. ${ }^{[25,26]}$ Poly(styrene-co-maleic anhydride) (PSMA) coating is achieved by mixing $2 \mathrm{~mL}$ of hybrid nanoparticles solution with PSMA (20 mg) in chloroform $(1 \mathrm{~mL})$ for $5 \mathrm{~h}$. Then ethanolamine $(20 \mu \mathrm{L})$ is added to the solution and mixed for 1-2 min. Next, recurring additions of TDW (1 mL) is done to transfer the particles to the above water phase followed by mild centrifugation before extraction. Polyethylenimine (PEI) coating is done by mixing HNPs solution $(1 \mathrm{~mL})$ with PEI (0.15 g; MW 25 000) in chloroform (1 mL) for $1 \mathrm{~h}$. Then the particles are precipitated and washed with cyclohexane (1:1 chloroform/cyclohexane), followed by centrifugation. TDW is added to the precipitate and residues of $\mathrm{PEI}$ are removed by centrifugation. In addition, both polymer-coated particles are washed again through $100 \mathrm{kDa}$ cellulose membrane, to remove polymer residues in the water suspended hybrids.

Nanoparticle Characterization: TEM and high resolution STEM characterization was performed using a Tecnai T12 G² Spirit and Tecnai $F 20 G^{2}$, respectively. All size statistics are done with "Scion image" program on 200 particles. Absorption was measured with a JASCO V-570 UV-vis-near IR spectrophotometer. Extinction coefficient values of the NPs were calculated using a previously reported method. ${ }^{[49]}$

Hydrogen Evolution Rate and Efficiency Measurements: In order to determine and measure the evolved hydrogen gas from the photocatalytic reaction using the HNP model systems, the following set-up is used. The photocatalysts were dispersed in TDW solution ( $2 \mathrm{~mL}$; optical density, OD 1 at $405 \mathrm{~nm}$ ). The photocatalyst solution was placed in a quartz cuvette and hole scavengers, $\mathrm{Na}_{2} \mathrm{~S}-9 \mathrm{H}_{2} \mathrm{O}$ and $\mathrm{Na}_{2} \mathrm{SO}_{3}$, (typically $0.05 \mathrm{M}$ and $0.07 \mathrm{M}$ respectively), were added to the water. The solution is purged with argon for $20 \mathrm{~min}$ and stirred. The HNPs were then illuminated with $40 \mathrm{~mW} 405 \mathrm{~nm}$ laser. Aliquots of the reaction vessel head space were taken using a gas tight syringe at different time intervals and detected and quantified using Varian gas chromatograph (model 6820) equipped with a molecular sieve ( $5 \AA$ ) packed column and a thermal conductivity detector. The resulting chromatograms and hydrogen concentration are obtained by the comparison to a calibration curve of known hydrogen amounts.

Transient Absorption Measurements: The laser system employed for ultrafast transient absorption was based on a TiSapphire chirped pulse amplified source, with maximum output energy of about $800 \mu \mathrm{J}, 1 \mathrm{kHz}$ repetition rate, central wavelength of $800 \mathrm{~nm}$ and pulse duration of about $150 \mathrm{fs}$. Excitation pulses 
at $400 \mathrm{~nm}$ were obtained by doubling the fundamental frequency in a $\beta$-Barium borate (BBO) crystal while other pump photons at different wavelength were generated by non-collinear optical parametric amplification in BBO, with pulse duration around $100 \mathrm{fs}$. Pump pulses were focused to $175 \mu \mathrm{m}$ diameter spot. Probing was achieved in the visible range by using white light generated in a thin sapphire plate, and in the UV-visible range by using a thin Calcium Fluoride plate. Chirp-free transient transmission spectra were collected by using a fast optical multichannel analyzer (OMA) with dechirping algorithm. The measured quantity is the normalized transmission change, $\Delta \mathrm{T} / \mathrm{T}$.

Fluorescence Spectra and Lifetime Measurements: Fluorescence emission measurements were performed with a Varian Cary Eclipse fl uorometer. Fluorescence lifetime measurements were performed using time-correlated single photon counting (TCSPC) using an Edinburgh Instruments FLS920 fluorometer with a TCC900 TCSPC card. Cuvettes containing solutions of CdSe/CdS seeded nanorods with different surface coatings were excited at a wavelength of $405 \mathrm{~nm}$ by an EPL-405 picosecond pulsed diode laser, with pulse width of $80 \mathrm{ps}$ and repetition rate of $1 \mathrm{MHz}$.

\section{Acknowledgement}

The research leading to these results has received funding from the European Research Council under the European Union's Sev-enth Framework Programme (FP7/2007-2013) / ERC grant agree-ment no. 246841, and by the Ministry of Science and Technology, Israel \& The Directorate General for Political and Security Affairs of the Ministry of Foreign Affairs, Italy. U.B. thanks the Alfred \& Erica Larisch memorial chair. G.C. acknowledges support by the EC under Graphene Flagship (contract no. CNECT-ICT-604391).Y.B.S. is grateful for a fellowship given by Israel Ministry of Science and Technology and Keren Hayesod Appeal. N.W was supported by a Clara Robert Einstein Scholarship.

[1] P. D. Yang, MRS Bull. 2012, 37, 806-813.

[2] K. Maeda, K. Domen, J. Phys. Chem. Lett. 2010, 1, 2655-2661.

[3] Z. J. Han, F. Qiu, R. Eisenberg, P. L. Holland, T. D. Krauss, Science 2012, 338, 1321-1324.

[4] R. Costi, A. E. Saunders, U. Banin, Angew. Chem. Int. Ed. 2010, 49, 4878-4897.

[5] A. Kudo, Y. Miseki, Chem. Soc. Rev. 2009, 38, 253-278.

[6] H. M. Zhu, N. H. Song, H. J. Lv, C. L. Hill, T. Q. Lian, J. Am. Chem. Soc. 2012, 134, 11701-11708.

[7] M. B. Wilker, K. J. Schnitzenbaumer, G. Dukovic, Israel J. Chem. 2012, 52, 1002-1015.

[8] U. Banin, Y. Ben-Shahar, K. Vinokurov, Chem. Mater. 2013, 26, 97-110.
[9] L. Amirav, A. P. Alivisatos, J. Phys. Chem. Lett. 2010, 1, 1051-1054.

[10] K. P. Acharya, R. S. Khnayzer, T. O’Connor, G. Diederich, M. Kirsanova, A. Klinkova, D. Roth, E. Kinder, M. Imboden, M. Zamkov, Nano Lett. 2012, 12, 522-522.

[11] N. Z. Bao, L. M. Shen, T. Takata, K. Domen, Chem. Mater. 2008, 20, 110-117.

[12] Y. Shemesh, J. E. Macdonald, G. Menagen, U. Banin, Angew. Chem. Int. Ed. 2011, 50, 1185-1189.

[13] M. Berr, A. Vaneski, A. S. Susha, J. Rodriguez-Fernandez, M. Doblinger, F. Jackel, A. L. Rogach, J. Feldmann, Appl. Phys. Lett. 2010, 97, 093108.

[14] M. L. Tang, D. C. Grauer, B. Lassalle-Kaiser, V. K. Yachandra, L. Amirav, J. R. Long, J. Yano, A. P. Alivisatos, Angew. Chem. Int. Ed. 2011, 50, 10203-10207.

[15] K. Maeda, K. Domen, Top. Curr. Chem. 2011, 303, 95-119.

[16] K. A. Brown, S. Dayal, X. Ai, G. Rumbles, P. W. King, J. Am. Chem. Soc. 2010, 132, 9672-9680.

[17] B. L. Greene, C. A. Joseph, M. J. Maroney, R. B. Dyer, J. Am. Chem. Soc. 2012, 134, 11108-11111.

[18] P. V. Kamat, J. Phys. Chem. Lett. 2012, 3, 663-672.

[19] T. O’Connor, M. S. Panov, A. Mereshchenko, A. N. Tarnovsky, R. Lorek, D. Perera, G. Diederich, S. Lambright, P. Moroz, M. Zamkov, ACS Nano 2012, 6, 8156-8165.

[20] K. F. Wu, H. M. Zhu, Z. Liu, W. Rodriguez-Cordoba, T. Q. Lian, J. Am. Chem. Soc. 2012, 134, 10337-10340.

[21] M. J. Berr, A. Vaneski, C. Mauser, S. Fischbach, A. S. Susha, A. L. Rogach, F. Jackel, J. Feldmann, Small 2012, 8, 291297.

[22] L. Carbone, A. Jakab, Y. Khalavka, C. Sonnichsen, Nano Lett. 2009, 9, 3710-3714.

[23] G. Menagen, J. E. Macdonald, Y. Shemesh, I. Popov, U. Banin, J. Am. Chem. Soc. 2009, 131, 17406-17411.

[24] W. C. W. Chan, S. M. Nie, Science 1998, 281, 2016-2018.

[25] T. Nann, Chem. Commun. 2005, 1735-1736.

[26] E. E. Lees, T. L. Nguyen, A. H. A. Clayton, P. Mulvaney, B. W. Muir, ACS Nano 2009, 3, 1121-1128.

[27] L. Carbone, C. Nobile, M. De Giorgi, F. D. Sala, G. Morello, P. Pompa, M. Hytch, E. Snoeck, A. Fiore, I. R. Franchini, M. Nadasan, A. F. Silvestre, L. Chiodo, S. Kudera, R. Cingolani, R. Krahne, L. Manna, Nano Lett. 2007, 7, 2942-2950.

[28] D. V. Talapin, J. H. Nelson, E. V. Shevchenko, S. Aloni, B. Sadtler, A. P. Alivisatos, Nano Lett. 2007, 7, 2951-2959.

[29] A. E. Saunders, I. Popov, U. Banin, J. Phys. Chem. B 2006, 110(50), 25421-25429.

[30] T. Mokari, E. Rothenberg, I. Popov, R. Costi, U. Banin, Science 2004, 304 (5678), 1787-1790.

[31] O. Varnavski, G. Ramakrishna, J. Kim, D. Lee, T. Goodson, J. Am. Chem. Soc. 2010, 132, 16-17.

[32] N. Waiskopf, R. Rotem, I. Shweky, L. Yedidya, H. Soreq, U. Banin, BioNanoScience 2013, 3, 1-11.

[33] N. Waiskopf, I. Shweky, I. Lieberman, U. Banin, H. Soreq, ACS Chem. Neurosci. 2011, 2 (3), 141-150.

[34] M. Shim, A. Javey, N. W. S. Kam, H. J. Dai, J. Am. Chem. Soc. 2001, 123, 11512-11513.

[35] A. M. Smith, H. W. Duan, M. N. Rhyner, G. Ruan, S. M. Nie, Phys. Chem. Chem. Phys. 2006, 8, 3895-3903.

[36] J. Aldana, Y. A. Wang, X. G. Peng, J. Am. Chem. Soc. 2001, 123, 8844-8850.

[37] D. Mongin, E. Shaviv, P. Maioli, A. Crut, U. Banin, N. Del Fatti, F. Vallee, ACS Nano 2012, 6, 7034-7043.

[38] T. S. Ahmadi, S. L. Logunov, M. A. El-Sayed, J. Phys. Chem. 1996, 100, 8053-8056.

[39] E. Khon, A. Mereshchenko, A. N. Tarnovsky, K. Acharya, A. Klinkova, N. N. Hewa-Kasakarage, I. Nemitz, M. Zamkov, Nano Lett. 2011, 11, 1792-1799. 
[40] P. Yu, X. Wen, Y.-C. Lee, W.-C. Lee, C.-C. Kang, J. Tang, J. Phys. Chem. Lett. 2013, 4, 3596-3601.

[41] J. He, W. Ji, G. H. Ma, S. H. Tang, E. S. W. Kong, S. Y. Chow, X. H. Zhang, Z. L. Hua, J. L. Shi, J. Phys. .Chem B 2005, 109, 4373-4376.

[42] A. Sitt, F. Della Sala, G. Menagen, U. Banin, Nano Lett. 2009, 9, 3470-3476.

[43] K. Wu, W. E. Rodríguez-Córdoba, Z. Liu, H. Zhu, T. Lian, ACS Nano 2013, 7, 7173-7185.

[44] A. Thibert, F. A. Frame, E. Busby, M. A. Holmes, F. E. Osterloh, D. S. Larsen, J. Phys. Chem. Lett. 2011, 2, 2688-2694.

[45] J. Tang, K. W. Kemp, S. Hoogland, K. S. Jeong, H. Liu, L. Levina, M. Furukawa, X. Wang, R. Debnath, D. Cha, K. W. Chou, A. Fischer,
A. Amassian, J. B. Asbury, E. H. Sargent, Nat. Mater. 2011, 10, 765-771.

[46] R. Zhou, R. Stalder, D. Xie, W. Cao, Y. Zheng, Y. Yang, M. Plaisant, P. H. Holloway, K. S. Schanze, J. R. Reynolds, J. Xue, ACS Nano 2013, 7, 4846-4854.

[47] W. W. Yu, X. G. Peng, Angew. Chem. Int. Ed. 2002, 41, 2368-2371.

[48] G. Menagen, D. Mocatta, A. Salant, I. Popov, D. Dorfs, U. Banin, Chem. Mater. 2008, 20, 6900-6902.

[49] E. Shaviv, A. Salant, U. Banin, ChemPhysChem 2009, 10, 1028-1031. 